\title{
Implications of $\operatorname{Br}(\mu \rightarrow e \gamma)$ and $\Delta a_{\mu}$ on muonic lepton flavour violating processes
}

\author{
Chun-Khiang CHUA* \\ Chung Yuan Christian University \\ E-mail: ckchuaecycu.edu.tw
}

\begin{abstract}
We study the implications of the experimental results on the $\mu \rightarrow e \gamma$ decay rate and the muon anomalous magnetic moment, on muonic lepton flavor violating processes, such as $\mu \rightarrow 3 e$ and $\mu N \rightarrow e N$. We use a model-independent approach in this analysis, where these processes are considered to be loop induced by exchanging spin- $1 / 2$ and spin- 0 particles. We explore two complementary cases-those with no cancellation mechanism in amplitudes and those with an internal (built-in) cancellation mechanism. Our main results are as follows: (a) Bounds from rates are used to constrain parameters, such as coupling constants and masses. These constraints can be easily updated by simple scalings, if the experimental situations change. (b) The muon $g-2$ data favor nonchiral interactions. (c) In $\mu \rightarrow 3 e$ and $\mu N \rightarrow e N$ processes, Z-penguin diagrams may play some role, while box diagram contributions to $\mu \rightarrow 3 e$ are usually highly constrained. (d) In the first case (without any built-in cancellation mechanism), using the recent $\mu \rightarrow e \gamma$ bound, we find that $\mu \rightarrow 3 e$ and $\mu N \rightarrow e N$ rates are usually bounded below the present experimental limits by two to three orders of magnitude in general. Furthermore, by comparing $\Delta a_{\mu}$ and $\mathscr{B}(\mu \rightarrow$ $e \gamma$ ) data, the couplings of $\mu$ and $e$ are found to be highly hierarchical. Additional suppression mechanisms should be called for. (e) In the second case (with a built-in cancellation mechanism), mixing angles can provide additional suppression factors to satisfy the $\Delta a_{\mu}$ and $\mathscr{B}(\mu \rightarrow e \gamma)$ bounds. While the $\mu \rightarrow 3 e$ rate remains suppressed, the bounds on $\mu N \rightarrow e N$ rates, implied from the latest $\mu \rightarrow e \gamma$ bound, can be relaxed significantly and can be just below the present experimental limits.
\end{abstract}

The European Physical Society Conference on High Energy Physics -EPS-HEP2013

18-24 July 2013

Stockholm, Sweden

* Speaker. 


\section{Introduction}

Charge lepton flavor violating (LFV) processes are prohibited in the Standard Model (SM) and, hence, are excellent probes of New Physics (NP). Recently the search of $\mu \rightarrow e \gamma$ decay was reported by the MEG collaboration giving [1] $\mathscr{B}\left(\mu^{+} \rightarrow e^{+} \gamma\right) \leq 5.7 \times 10^{-13}$. The bound is several times lower than the previous one [2,3]. In many New Physics models this decay mode is closely related to other lepton flavor violating processes, such as $\mu^{+} \rightarrow e^{+} e^{+} e^{-}$decays and $\mu^{-} N \rightarrow e^{-} N$ conversions [4]. The present limits and future experimental sensitivities [1, 3, 5] of these LFV processes are summarized in Table 1 . Note that present bounds on $\mu$ LFV rates are roughly of similar orders. It will be interesting to see what the implications are of the new $\mathscr{B}(\mu \rightarrow e \gamma)$ bound on these LFV processes and the interplay between them [6].

Since 2001, the muon anomalous magnetic moment remains as a hint of a NP contribution (see, for a review, [7]). Experimental data deviates from the Standard Model (SM) expectation by more than $3 \sigma$ [3]: $\Delta a_{\mu}=a_{\mu}^{\text {exp }}-a_{\mu}^{\mathrm{SM}}=(287 \pm 63 \pm 49) \times 10^{-11}$. Since NP contributes to $\Delta a_{\mu}$ and $\mathscr{B}\left(\mu^{+} \rightarrow e^{+} \gamma\right)$ through very similar loop diagrams [see Fig. 1(a) and (b)], it is useful to compare them at the same time.

We consider a class of models that muon $g-2$ and various muon lepton flavor violating processes, such as $\mu \rightarrow e \gamma, \mu \rightarrow 3 e$ and $\mu \rightarrow e$ conversions, are loop-induced by exchanging spin-1/2 and spin-0 particles [6]. We try to see where the present $g-2$ and $\mu \rightarrow e \gamma$ experimental results lead us on estimating rates or bounds on various LFV muonic decay modes and the interplay between them. Two cases, which are complementary to each other, are considered. In the first case (Case I), there is no built-in cancellation mechanism among amplitudes. The second case (Case II) is with some built-in mechanism, such as GIM or super GIM mechanism.

\section{Results: Case I}

We start with Case I. In Fig. 2 we show the allowed parameter space for $Q_{\phi, \psi}\left|g_{\mu L(R)}\right|^{2} / m_{\psi}^{2}$ and $Q_{\phi, \psi} \operatorname{Re}\left(g_{\mu R}^{*} g_{\mu L}\right) / m_{\psi}\left(g_{l L(R)}\right.$ are couplings of $\phi-\psi-l_{L(R)}$ interactions) constrained by the measured $\Delta a_{\mu}$ with exclusion of $\left|g_{\mu L(R)}\right|^{2},\left|g_{\mu L} g_{\mu R}\right|>4 \pi$ and $m_{\psi, \phi}<100 \mathrm{GeV}$. The latter requirements are to ensure perturbativity and to satisfy the experimental bounds on the masses of exotic particles [3]. Bands denoted with $\phi$ or $\psi$ are allowed regions obtained through contributions from diagrams with $\phi$ or $\psi$ interacting with a photon [see Fig 1(a) and (b)]. From Fig. 2(a), we see that the allowed regions on $Q_{\phi}\left|g_{\mu L(R)}\right|^{2} / m_{\psi}^{2}$ and $-Q_{\psi}\left|g_{\mu L(R)}\right|^{2} / m_{\psi}^{2}$ are similar and the signs of $Q_{\phi, \psi}$ are constrained by data. Note that the allowed parameter space is quite limited and is almost closed by the bounds

Table 1: Current experimental upper limits and future sensitivities on various $\mu$ LFV processes $[1,3,5]$.

\begin{tabular}{lcc}
\hline & current limit & future sensitivity \\
\hline $\mathscr{B}\left(\mu^{+} \rightarrow e^{+} \gamma\right)$ & $<5.7 \times 10^{-13}$ & $10^{-13}$ \\
$\mathscr{B}\left(\mu^{+} \rightarrow e^{+} e^{+} e^{-}\right)$ & $<1.0 \times 10^{-12}$ & $10^{-14}-10^{-16}$ \\
$\mathscr{B}\left(\mu^{-} \mathrm{Ti} \rightarrow e^{-} \mathrm{Ti}\right)$ & $<4.3 \times 10^{-12}$ & $10^{-18}$ \\
$\mathscr{B}\left(\mu^{-} \mathrm{Au} \rightarrow e^{-} \mathrm{Au}\right)$ & $<7 \times 10^{-13}$ & $10^{-14}-10^{-16}$ \\
$\mathscr{B}\left(\mu^{-} \mathrm{Al} \rightarrow e^{-} \mathrm{Al}\right)$ & $\ldots$ & $10^{-16}$ \\
\hline
\end{tabular}




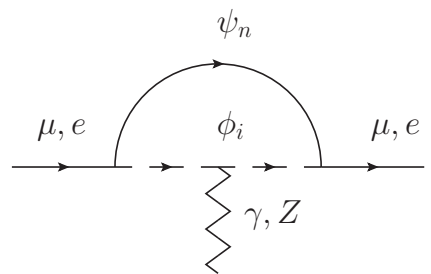

(a)

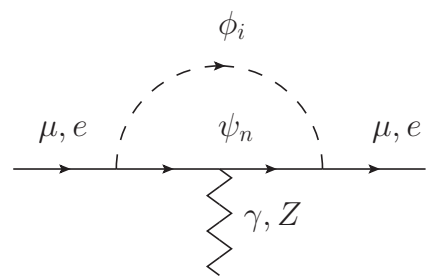

(b)

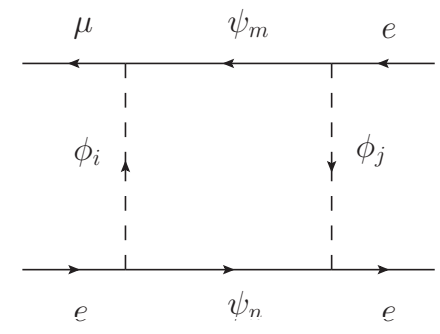

(c)

Figure 1: (a) and (b): Penguin diagrams that contribute to muon $g-2, \mu^{+} \rightarrow e^{+} \gamma, \mu^{+} \rightarrow e^{+} e^{+} e^{-}$and $\mu^{-} N \rightarrow e^{-} N$ processes. Note that diagrams involving self energy parts are not shown. (c) : Box diagram contributing to the $\mu^{+} \rightarrow 3 e$ process. Another diagram is needed when $\psi_{m, n}$ are Majorana fermions.

from $\left|g_{\mu L(R)}\right|^{2}<4 \pi$ and $m_{\psi}>100 \mathrm{GeV}$ and $m_{\phi}>100 \mathrm{GeV}$. Thus, it is unlikely to use a chiral-type interaction to generate the measured $\Delta a_{\mu}$. From Fig. 2(b), we see that the allowed parameter space is substantially larger. We now turn to $\mu \mathrm{LFV}$ processes, including $\mu \rightarrow e \gamma, \mu \rightarrow 3 e, \mu \mathrm{Ti} \rightarrow e \mathrm{Ti}$ and $\mu \mathrm{Au}(\mathrm{Al}) \rightarrow e \mathrm{Au}(\mathrm{Al})$ transitions. In Fig. 2(c) and (d), we show the parameter space excluded by various bounds and the one corresponding to projections from the expected sensitivities on these $\mu$ LFV processes, through contributions from photonic diagrams. In Fig. 2(e) and (f) rate ratios of various modes through photonic penguins are given. Since the present experimental bounds on LFV rates are of similar orders of magnitudes (see Table 1), the present bound on the $\mu \rightarrow e \gamma$ rate provides the most severe constraint. We find that $\mu \rightarrow 3 e$ and $\mu^{-} N \rightarrow e^{-} N$ rates are bounded below the present experimental limits by two to three orders of magnitude in general.

Since NP contributions to $\Delta a_{\mu}$ and the $\mu^{+} \rightarrow e^{+} \gamma$ decay are from similar diagrams, it will be useful to compare them. We find that the present data on $\Delta a_{\mu}$ and $\mathscr{B}\left(\mu^{+} \rightarrow e^{+} \gamma\right)$ lead to $\frac{g_{\mu R(L)} g_{e L(R)}}{g_{\mu R} g_{\mu L}}=\frac{g_{e L(R)}}{g_{\mu L(R)}} \leq 2.1 \times 10^{-5} \simeq \lambda^{7}$, where we define $\lambda \equiv 0.2$. This ratio is much smaller than any known coupling ratio and mixing angle among the first and second generations. It seems that the present case is unnatural.

\section{Results: Case II}

We now turn to Case II. Results are shown in Fig. 3 (note that $\delta$ is a mixing angle of $\phi$ ). Fig. 3(a) is same as Fig. 2(a). Comparing Fig. 3(b) to Fig. 2(b), we see that the allowed parameters in the upper $m_{\phi} / m_{\psi}$ region are similar. In contrast, they are relaxed substantially in the lower $m_{\phi} / m_{\psi}$ region in the present case. The built-in cancellation mechanism reduces the amplitudes effectively and a too heavy $\psi$ is incapable to produce a large enough $\Delta a_{\mu}$. The effect of the cancellation is important in the low $m_{\phi} / m_{\psi}$ region and, consequently, relaxes the constraints on parameters. In Fig. 3(c) and (d), we show the constrained and projected parameter space through penguin contributions by considering the experimental bounds and the proposed sensitivities. By comparing the constraints from $\Delta a_{\mu}$ and $\mathscr{B}(\mu \rightarrow e \gamma)$ we obtain $\frac{g_{\mu R(L)} g_{e L(R)} \operatorname{Re}\left[\left(\delta_{R L(L R)}\right)_{\mu e}\right]}{g_{\mu R} g_{\mu L} \operatorname{Re}\left[\left(\delta_{R L}\right)_{\mu \mu}\right]}=\frac{g_{e L(R)}}{g_{\mu L(R)}} \frac{\operatorname{Re}\left[\left(\delta_{R L(L R)}\right)_{\mu e}\right]}{\operatorname{Re}\left[\left(\delta_{R L}\right)_{\mu \mu}\right]} \leq$ $2.1 \times 10^{-5} \simeq \lambda^{7}$. If we estimate $g_{e L(R)} / g_{\mu L(R)}$ by using the lepton mass ratio $m_{e} / m_{\mu} \sim \lambda^{3 \sim 4}$, we see that a mixing angle ratio of $\operatorname{Re}\left[\left(\delta_{R L(L R)}\right) \mu e\right] / \operatorname{Re}\left[\left(\delta_{R L}\right)_{\mu \mu}\right] \lesssim \lambda^{3 \sim 4}$, which is not unnatural, can easily satisfy the above bound. In this respect, case II is more reasonable and natural than case I, where the 


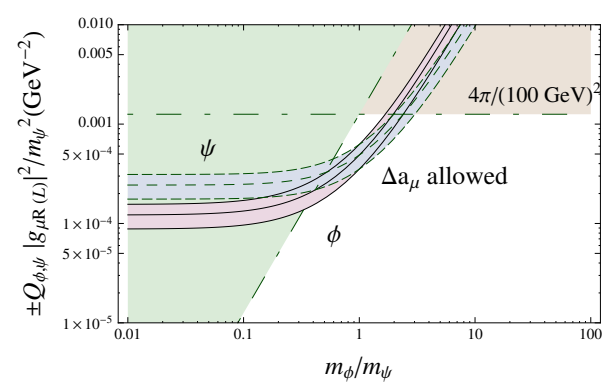

(a)

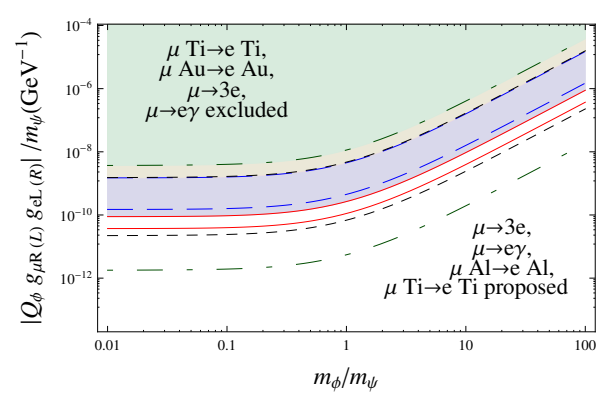

(c)

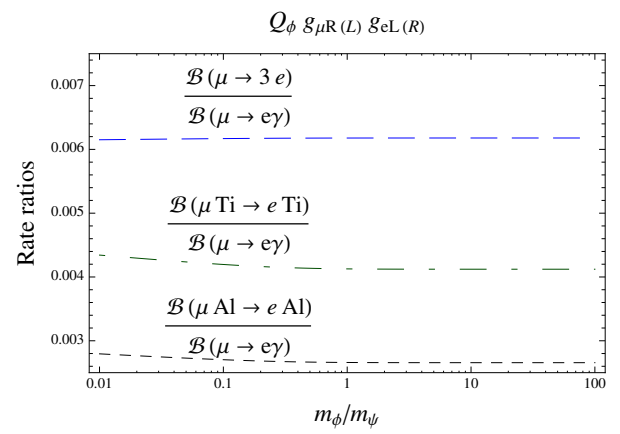

(e)

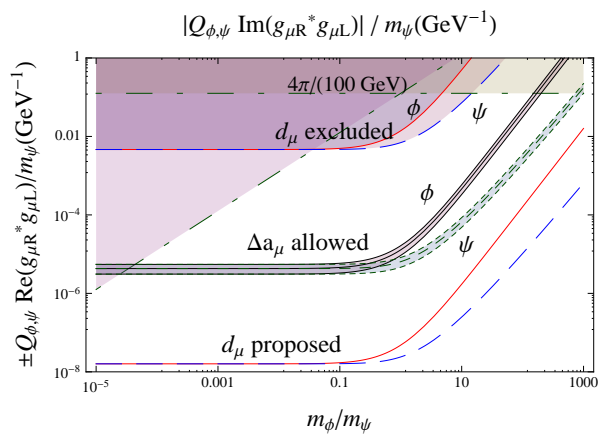

(b)

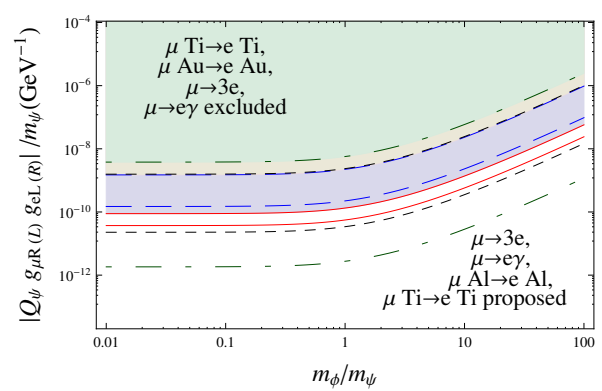

(d)

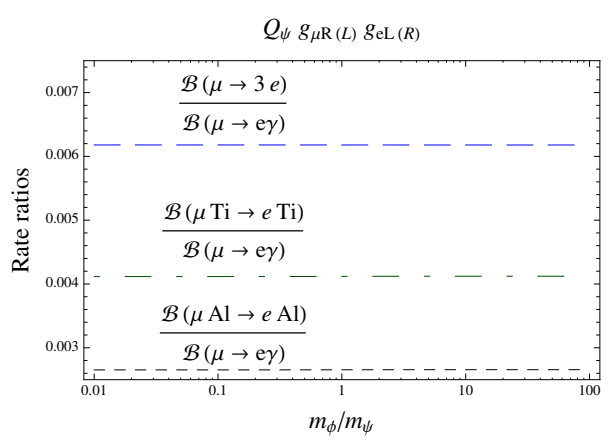

(f)

Figure 2: (a), (b): Allowed parameter space for $\pm Q_{\phi, \psi}\left|g_{\mu L(R)}\right|^{2} / m_{\psi}^{2}$ and $\pm Q_{\phi, \psi} \operatorname{Re}\left(g_{\mu R}^{*} g_{\mu L}\right) / m_{\psi}$ constrained by $\Delta a_{\mu}$. (c), (d): Parameter space excluded (projected) by various bounds (expecting sensitivities) on $\mu$ LFV processes through contributions from photonic penguins. (e), (f): Ratios of rates contributed from photonic penguins.

coupling ratio is highly hierarchical. It is interesting to see from Fig. 3 (c) and (d) that the $\mu \rightarrow e \gamma$ bound is not always the most stringent one. For example, comparing Fig. 3(c) with Fig. 2(c), we see that in the low $m_{\phi} / m_{\psi}$ region both bounds from $\mu \rightarrow e \gamma$ and $\mu \rightarrow 3 e$ are relaxed up to three orders of magnitude, while the changes on those from $\mu N \rightarrow e N$ are mild. We can infer that, similar to the $\Delta a_{\mu}$ case, the $F_{2}$ (photonic) penguin amplitudes exhibit cancellations in amplitudes in the low $m_{\phi} / m_{\psi}$ region and relax the constraints from $\mu \rightarrow e \gamma$ significantly, while the cancellations in the $F_{1}$ penguin contributions in $\mu \rightarrow 3 e$ and $\mu N \rightarrow e N$ processes are mild. As a result the bounds from $\mu N \rightarrow e N$ approach the $\mu \rightarrow e \gamma$ bound in this case, while in the previous case these two bounds are always apart. The ratios of photonic penguin contributing rates plotted in Fig. 3(e) and (f) show 


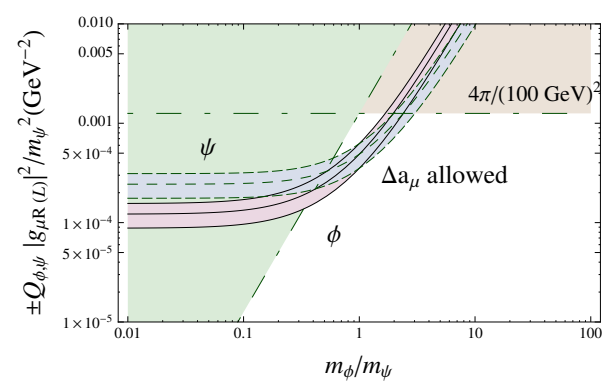

(a)

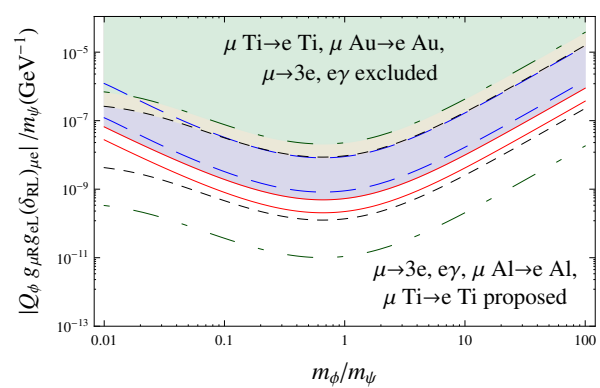

(c)

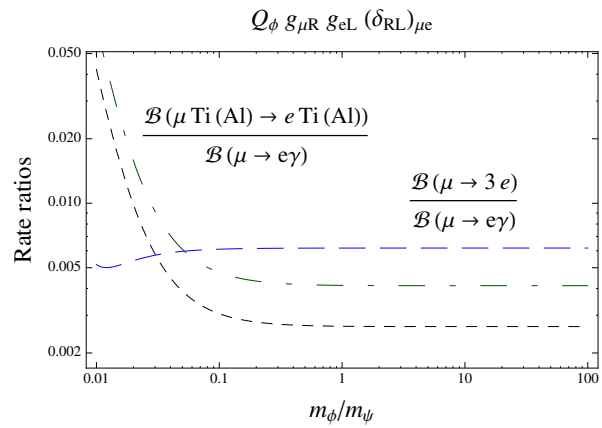

(e)

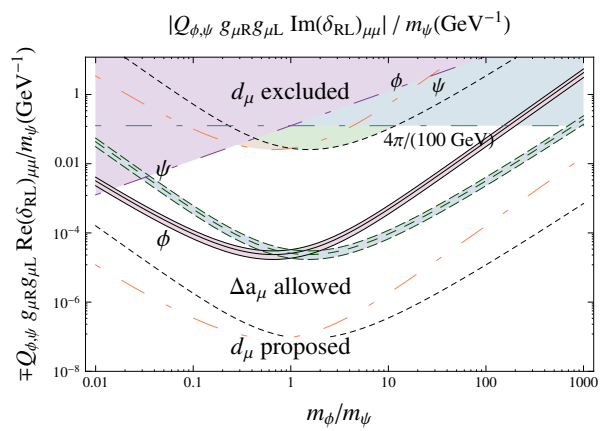

(b)

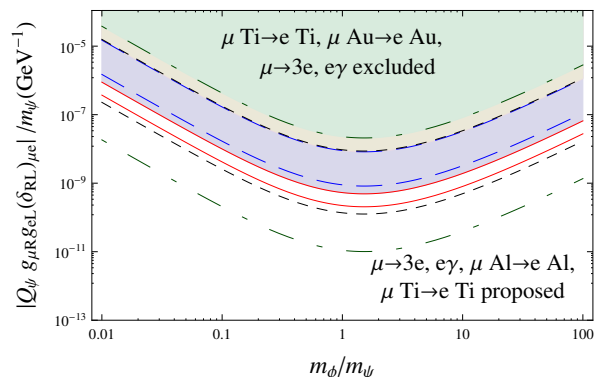

(d)

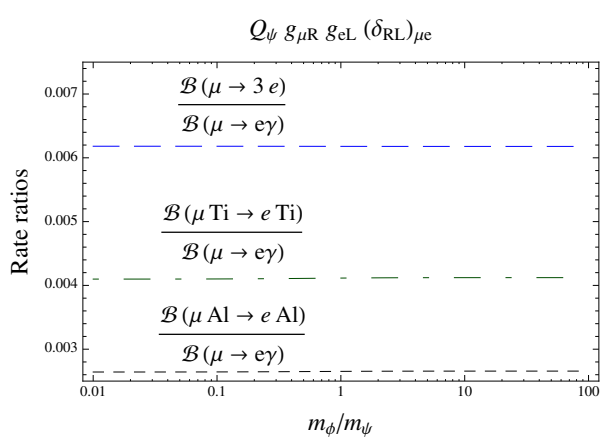

(f)

Figure 3: Same as Fig. 2, but in Case II.

that $\mathscr{B}(\mu N \rightarrow e N) / \mathscr{B}(\mu \rightarrow e \gamma)$ and $\mathscr{B}(\mu \rightarrow 3 e) / \mathscr{B}(\mu \rightarrow e \gamma)$ are enhanced compared with those in Fig. 2(e) and (f). In Fig. 3(e) the $\mathscr{B}(\mu N \rightarrow e N) / \mathscr{B}(\mu \rightarrow e \gamma)$ ratio is enhanced by one order of magnitude, while the $\mathscr{B}(\mu \rightarrow 3 e) / \mathscr{B}(\mu \rightarrow e \gamma)$ ratio does not change much. It is very interesting that the rate ratio $\mathscr{B}(\mu N \rightarrow e N) / \mathscr{B}(\mu \rightarrow e \gamma)$ from the $g_{\mu R(L)} g_{e L(R)}$ term is enhanced and different from Case I. We find that the present bound on $\mu \rightarrow e \gamma$ allows $\mathscr{B}(\mu N \rightarrow e N) \lesssim 10^{-14}$, which is close to the present bounds (see Table 1). Therefore, the search on these processes could be very interesting. In Fig. 4, we show the constraints on parameters which contribute through box diagrams to the $\mu^{+} \rightarrow 3 e$ process in this case. Note that constraints on the same combinations of parameters can be obtained from penguin processes, including $\mu \rightarrow e \gamma, \Delta a_{e}$, EDM, and the purturbative bounds, as well. We see that these constraints are usually much stronger than the ones from the box diagrams. Hence, in general, these box diagrams do not play a major role in the $\mu^{+} \rightarrow 3 e$ decay. Results from $Z$ exchange can be found in Ref. [6]. 


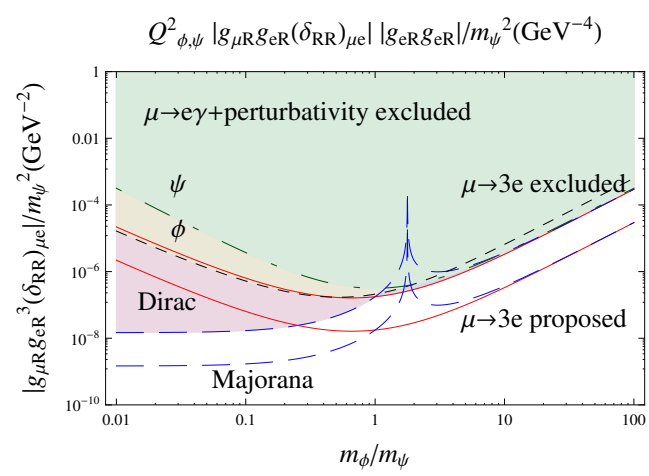

(a)

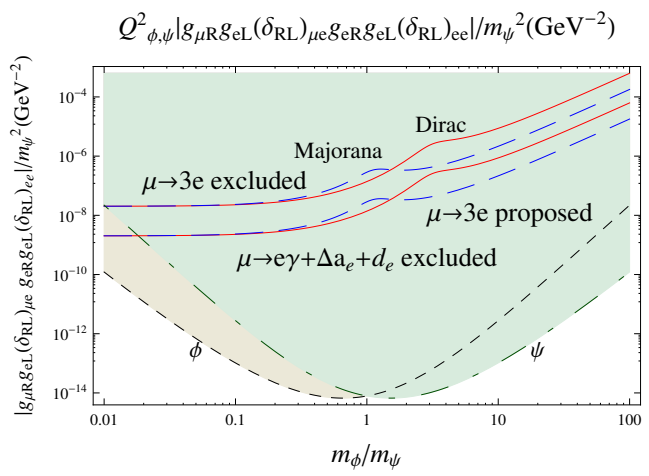

(b)

Figure 4: Constraints on parameters which contribute through box diagrams to the $\mu \rightarrow 3 e$ process.

\section{Conclusion}

In conclusion, we use a model independent approach in this analysis, where these processes are considered to be loop-induced by exchanging spin-1/2 and spin-0 particles [6]. We explore two complementary cases, without or with an internal (built-in) cancellation mechanism in amplitudes. Our main results are as follows: (a) Bounds from rates are used to constrain parameters, such as coupling constants and masses. (b) The muon $g-2$ data favor nonchiral interactions. (c) In $\mu \rightarrow 3 e$ and $\mu^{-} N \rightarrow e^{-} N$ processes, the $Z$-penguin diagrams may play some role, while the box diagram contributions to the $\mu \rightarrow 3 e$ rate are usually highly constrained. (d) In the first case (without any built-in cancellation mechanism), using the recent $\mu^{+} \rightarrow e^{+} \gamma$ bound, we find that $\mu \rightarrow 3 e$ and $\mu^{-} N \rightarrow e^{-} N$ rates are bounded below the present experimental limits by two to three orders of magnitude in general. Furthermore, by comparing $\Delta a_{\mu}$ and $\mathscr{B}(\mu \rightarrow e \gamma)$ data, the couplings of $g_{\mu}$ and $g_{e}$ are found to be highly hierarchical. Additional suppression mechanisms should be called for. (e) In the second case (with a built-in cancellation mechanism), mixing angles can provide additional suppression factors to satisfy the $\Delta a_{\mu}$ and $\mathscr{B}(\mu \rightarrow e \gamma)$ bounds without relaying only on highly hierarchical $g_{e}$ and $g_{\mu}$ couplings. In addition, although the $\mu \rightarrow 3 e$ rate remains suppressed, the bounds on $\mu^{-} N \rightarrow e^{-} N$ rates, implicated by the MEG $\mu^{+} \rightarrow e^{+} \gamma$ bound, can be relaxed significantly in the second case and can be just below the present experimental limits.

\section{References}

[1] J. Adam et al. [MEG Collaboration], Phys. Rev. Lett. 110, 201801 (2013).

[2] J. Adam et al. [MEG Collaboration], Phys. Rev. Lett. 107, 171801 (2011).

[3] J. Beringer et al. [Particle Data Group Collaboration], Phys. Rev. D 86, 010001 (2012).

[4] Y. Kuno and Y. Okada, Rev. Mod. Phys. 73, 151 (2001).

[5] Y. Kuno, PoS FPCP 2010, 049 (2010); M. De Gerone and M. E. G. Collaboration, arXiv:1108.2670; T. Nomura, talk given at FPCP 2011; B. Casey, ibid.; A. Hoecker, arXiv:1201.5093.

[6] C. -K. Chua, Phys. Rev. D 86, 093009 (2012).

[7] F. Jegerlehner and A. Nyffeler, Phys. Rept. 477, 1 (2009). 